\title{
Valeurs de la République et islam à Mayotte
}

Mathilde Philip-Gay

\section{OpenEdition}

\section{Journals}

Édition électronique

URL : http://journals.openedition.org/rdr/329

DOI : $10.4000 /$ rdr.329

ISSN : 2534-7462

\section{Éditeur}

Presses universitaires de Strasbourg

\section{Édition imprimée}

Date de publication : 6 novembre 2018

Pagination : 59-76

ISBN : 979-10-344-0023-2

ISSN : 2493-8637

\section{Référence électronique}

Mathilde Philip-Gay, "Valeurs de la République et islam à Mayotte », Revue du droit des religions [En ligne], 6 | 2018, mis en ligne le 25 novembre 2019, consulté le 19 novembre 2020. URL : http:// journals.openedition.org/rdr/329; DOI : https://doi.org/10.4000/rdr.329

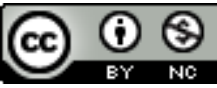

La revue du droit des religions est mise à disposition selon les termes de la Creative Commons Attribution - Pas d'Utilisation Commerciale 4.0 International - CC BY-NC 4.0. 


\section{VALEURS DELA RÉPUBLIQUEETISLAM ÀMAYOTTE}

\section{Mathilde PHILIP-GAY}

Université Jean Moulin Lyon 3, Équipe de droit public de Lyon (EDPL)

\section{RÉSUMÉ}

Mayotte cultive sa singularité, y compris cultuelle, puisque $95 \%$ de sa population est musulmane. Afin de préparer la départementalisation, le législateur a d'abord imposé les valeurs républicaines, par une transformation profonde du statut personnel de droit local et la suppression de la justice cadiale. Depuis, le département de Mayotte est intégré dans le plan national de formation aux valeurs de la République, et une nouvelle forme de conciliation entre valeurs universelles et valeurs singulières apparaît progressivement. Toutefois, pour éviter la déliquescence de ces valeurs, deux repères propres à cette société doivent être maintenus: les cadis, en tant que médiateurs sociaux, et son caractère matriarcal. Il est aussi nécessaire de garantir l'application à Mayotte de l'ensemble des principes constitutionnels.

\section{Abstract}

Mayotte, a French island located in the Indian Ocean, has always striven for maintaining its cultural identity and particularly its Islamic customary law. In order to prepare the department status, the legislator first imposed constitutional values, among others by changing the personal status and by suppressing the cadi courts. Since then, Mayotte has been integrated into the national plan for training in the French republican values and a new form of reconciliation between universal and singular values is gradually emerging. However, to avoid the failing of those singular values, priority attention needs to be given in ensuring cadi's key role as a mediator and, also, in providing support to the matriarchal tradition by giving women some special status. It is also necessary to ensure that all constitutional principles are implemented in Mayotte. 
E n 2010, le législateur prévoyait que Mayotte pourrait «conserver ce qui fait son identité dans la République ${ }^{1} »$.

Il est vrai que ce petit département de l'océan Indien ${ }^{2}$, faisant géographiquement partie de l'archipel des Comores, a toujours cultivé sa singularité. Déjà, alors que les invasions arabes $\mathrm{du}_{\mathrm{X} I I^{\mathrm{e}}}$ au XV $\mathrm{Xv}^{\mathrm{e}}$ siècle l'avaient placé sous la domination de sultans musulmans ${ }^{3}$, à la place des chefferies traditionnelles, la société a résisté fortement au nouveau modèle. Dans sa thèse de référence sur la vie quotidienne mahoraise, Sophie Blanchy explique cette résistance par la force des principes matrilinéaires (la filiation est définie dans la lignée maternelle) et de la matrilocalité (la résidence de la famille est celle de la mère) sur lesquels elle fonctionnait. Elle montre que cette opposition à certaines règles et principes venus d'ailleurs est encore aujourd'hui l'une des caractéristiques essentielles de la société mahoraise ${ }^{4}$, même si son modèle s'est finalement soumis à des influences malgaches, de l'Afrique de l'Est, arabo-musulmanes et françaises.

Mayotte se singularise aussi d'un point de vue cultuel depuis qu'elle a été vendue à la France ${ }^{5}$. Alors que sur le reste du territoire français l'islam est placé dans une position de culte minoritaire, actuellement $95 \%$ de la population mahoraise seraient de confession musulmane. Certes, une petite partie d'entre elle est chiite, mais elle se rattache principalement à l'école chaféite, avec une pratique empreinte de soufisme ${ }^{6}$, tout en intégrant des traditions animistes. Cet islam mahorais se caractérise par des cérémonies rassembleuses, telles ces célébrations musicales et chorégraphiques pouvant accompagner l'exercice du culte ${ }^{7}$, ou bien l'attachement à des fêtes, comme le "Grand mariage». C'est certainement cette identité très forte des pratiques mahoraises qui explique qu'aujourd'hui encore subsistent sur l'île un

1. L. n² 2010-1487, 7 déc. 2010, relative au département de Mayotte (exposé des motifs).

2. De $374 \mathrm{~km}^{2}$, il se compose de l'île de Grande-Terre, de Petite-Terre, et de plusieurs îlots.

3. F. HeRmet, «Regards croisés sur le dernier-né des départements français», in F. Hermet, Mayotte. État des lieux, enjeux et perspectives. Regards croisés sur le dernier-né des départements français, Paris, L'Harmattan, 2015, p. 13.

4. S. Blanchy, La vie quotidienne à Mayotte (Comores): essai d’anthropologie compréhensive. Anthropologie sociale et ethnologie, Université de la Réunion, 1988, spéc. p. 14 à 22.

5. Traité du 25 avril 1841, portant cession de Mayotte à la France, ratifié par le Roi le 10 février 1843.

6. Cela se traduit chaque semaine par l'organisation de cérémonies mêlant chants, danses et louanges (dont le Dahira Chadhouli et le Maoulida shengue, qui ont une importance particulière sur l'île).

7. V. Randrianary, Patrimoine musical et chorégraphique de Mayotte, Rapport de mission, Paris, Ministère de la Culture et de la Communication, 2007, p. 5. 
certain protectionnisme confessionnel et une véritable méfiance envers les interprétations religieuses, comportements et financements «de l'étranger ». Par exemple, en 2015, la mosquée de M'Tsangamouji, dont les prêches de l'imam étaient jugés trop radicaux, a été mise à sac par des habitants qui avaient pourtant œuvré à sa construction.

La singularité de Mayotte ne s'arrête pas à son modèle social ni à sa religion majoritaire. Son histoire est elle aussi très particulière, puisqu'elle est toujours «à contresens ${ }^{8} »$. Dans le «sillage des mouvements de libération nationale des années 1950-60, les autres îles des Comores ont opté pour l'indépendance», tandis que certaines collectivités de l'outre-mer français gagnaient en autonomie institutionnelle pour une meilleure prise en compte de leurs spécificités ${ }^{9}$. Mais Mayotte a fait l'inverse: son statut n'a fait qu'évoluer vers davantage d'intégration institutionnelle. Si bien qu'elle a été successivement: une colonie ${ }^{10}$, une collectivité territoriale ${ }^{11}$, une collectivité départementale ${ }^{12}$, une collectivité d'outre-mer «assimilée, à statut de base ${ }^{13}$ » avant d'accéder, à partir de 2011, au statut de département d'outre-mer (DOM) à compétences régionales. Ces étapes ont été marquées par la volonté de la population, plusieurs fois exprimée, de rester française. D'abord, en 1958, alors que les Comores avaient choisi de rester un territoire d'outre-mer (TOM), Mayotte aurait vainement souhaité devenir un DOM. La même scission entre Mayotte et le reste des Comores a été manifeste en 1974, lors du référendum d'autodétermination lorsque $65 \%$ de Mahorais ont voté contre l'indépendance, tandis que les Comoriens se sont prononcés à $95 \%$ pour l'autonomie. En 1975, par application du droit des peuples à disposer d'eux-mêmes, les Comores, nouvellement indépendantes, ont été admises à l'Organisation des Nations unies avec Mayotte, sans que cela ne suscite aucune réserve de la part de la France ${ }^{14}$ qui l'avait précisément dotée du statut de collectivité territoriale afin de favoriser, à terme, sa réintégration au sein des Comores ${ }^{15}$. Mais en 1976,

8. F. Riviere, « Préface», in F. Hermet, Mayotte..., op. cit., p. 11.

9. Ibid.

10. De 1841 à 1975. V. L.-A. BARRIERE, «Décolonisation, indépendance et droit de la nationalité dans l'océan Indien », in J. KNETSCH, E. RALSER, La nationalité française dans l'Océan indien, Paris, Société de législation comparée, 2015, p. 27-28.

11. De 1976 à 2000.

12. De 2001 à 2007.

13. O. Gohin, « Mayotte: la longue marche vers le droit commun », RJOI 2009, Numéro spécial Mayotte, p. 5 (V. aussi O. GoHIN, « Mayotte en droit constitutionnel français », in H. Bonin (dir.), Mayotte en France. Enjeux et tensions, Paris, Les Indes savantes, 2017, p. 49-65).

14. Elle ne participe pas au vote.

15. L. n 76-1212, 24 déc. 1976, relative à l'organisation de Mayotte. 
99,4 \% des votants à un nouveau référendum refusent encore une fois la réintégration ${ }^{16}$; une consultation légitimée par la décision du Conseil constitutionnel en vertu de laquelle Mayotte «ne saurait sortir de la République française sans le consentement de sa propre population», son appartenance à la République ne pouvant être constatée autrement qu'en droit constitutionnel français, et «nonobstant toute intervention d'une instance internationale ${ }^{17}$ ». Pourtant, s'est régulièrement posée, et se pose encore, la question de la compatibilité avec le droit international de cette prise en compte de la volonté de la population mahoraise. En effet, en principe, les résultats d'un tel référendum doivent être calculés sur l'ensemble du territoire, et non "îles par îles ${ }^{18}$ ", l'interprétation du principe de l'autodétermination des peuples respectant les frontières coloniales ${ }^{19}$. C'est en cela que la résolution 31/4 du 21 octobre 1976 a condamné, d'une part, les référendums «imposés aux habitants de l'île comorienne de Mayotte» et, d'autre part, la présence française. Ce qui n'a pas empêché que 95,24 \% des suffrages exprimés lors du référendum de 2009 soient favorables à la départementalisation.

Mayotte est donc entrée volontairement dans la République ${ }^{20}$ sans que soit posée la question de la conciliation de ses propres valeurs singulières avec les valeurs communes réaffirmées par la Constitution de 1958. Pourtant, comme le remarque Françoise Rivière,

au «moment où la société française place la laïcité au cœur du débat citoyen en lien avec les polémiques suscitées par la place de l'Islam et des autres composantes religieuses, la départementalisation de Mayotte et son statut de région européenne posent un autre défi à la République, celui d'être à la hauteur des valeurs qu'elle porte depuis le départ: la promesse d'une société pluriculturelle et pluricultuelle respectueuse des différences ${ }^{21} »$.

Pendant longtemps cette question a été éclipsée par d'autres, pour trois raisons principales. La première est que Mayotte, en dépit de ses atouts économiques ${ }^{22}$, est le département de France ayant la plus importante croissance

16. Résultats du scrutin d'autodétermination: JO 1976, p. 1625.

17. Cons. const., 30 déc. 1975, n 75-59 DC, Loi relative aux conséquences de l'autodétermination des îles des Comores.

18. AG ONU, rés. 3161 (XXVIII), 14 déc. 1973.

19. CIJ, Ch., 22 déc. 1986, Différend frontalier: Rec. CIJ 1986, p. 567.

20. Le $1^{\text {er }}$ janvier 2014, Mayotte a aussi accédé au statut de Région ultra périphérique de l'Union européenne.

21. F. RiviÈRE, art. cit., p. 11.

22. Par sa situation géographique, ses fonds marins, la culture d'épices, de vanille ou d'ylangylang, et son taux de natalité très élevé (V. M. Goujon, F. Hermet, « La pauvreté à 
démographique et étant le plus densément peuplé, avec une forte immigration irrégulière, un taux de chômage particulièrement élevé, et des flambées de violence ou d'insécurité $e^{23}$. Il a donc d'abord fallu - et il faut encore - l'équiper de structures administratives, sanitaires, sociales et scolaires; et ce sont ces problématiques qui l'ont initialement emporté sur celle des valeurs, alors qu'il aurait peut-être été nécessaire de les lier entre elles. De plus, d'après l'article 2 de la Constitution, «la langue de la République est le français», alors que celle de plus de $80 \%$ des Mahorais est le shimaoré (qui se rapproche du swahili parlé en Afrique orientale), voire le shibushi, aux racines malgaches; étant précisé que l'apprentissage de l'arabe est également très répandu pour des raisons religieuses, sans être une langue de la vie quotidienne. La question de l'application des valeurs de la République est donc également une question linguistique, qui implique de savoir comment traduire ces valeurs dans les langues de l'île, en s'appuyant sur des valeurs préexistantes.

Or dans une situation de possibles confrontations entre des valeurs communes et des valeurs singulières, Catherine Le Bris décrit deux modèles. Le premier est l'«ordre imposé», qui repose sur l'unité, et s'appuie sur «un droit de la subordination» pour imposer des valeurs communes. Le second modèle, l'«ordre négocié », repose sur la diversité et la construction d'un "en commun», condition du vivre ensemble ${ }^{24}$. La départementalisation de Mayotte semble avoir mis fin à la période de l'ordre imposé (1) pour passer à celle de l'ordre négocié (2).

\section{L'ORDRE IMPOSÉ JUSQU'À LA DÉPARTEMENTALISATION}

Par l'ordre imposé, l'État cherche à réaffirmer son monopole en imposant des valeurs communes à l'aide de deux instruments juridiques, que sont la loi et la justice. À Mayotte, cet ordre imposé est symbolisé par un changement de vocabulaire dans les textes normatifs. À partir de 2000, les renvois à «l'état civil des Comoriens musulmans ${ }^{25}$ » ou «au statut personnel coranique ${ }^{26}$ »

Mayotte: une évaluation par l'indice de la pauvreté humaine», in F. HERMET, Mayotte..., op.cit., p. 97).

23. V. Geisser, "Mayotte, si loin de Paris et pourtant si emblématique de nos "hypocrisies françaises" ", Migrations Société, n²016/2, p. 6.

24. C. LE BRIS, «La contribution du droit à la construction d'un "vivre ensemble" : entre valeurs partagées et diversité culturelle», Droit et société, n 92, 2016, p. 75.

25. Ord. $\mathrm{n}^{\circ}$ 2000-219, 8 mars 2000, relative à l'état civil à Mayotte, art. 4.

26. Ord. $n^{\circ} 77-450,29$ avril 1977, portant extension et adaptation aux communes de Mayotte du code des communes, art. 4 al. 2. 
disparaissent. L'appellation du statut personnel perd toute référence religieuse devenant «le statut civil de droit local applicable à Mayotte ${ }^{27}$ » (1.1). Cet ordre imposé s'est aussi manifesté par la suppression de la justice cadiale (1.2).

\subsection{LA TRANSFORMATION DU STATUT DE DROIT MUSULMAN EN UN STATUT «DE DROIT LOCAL»}

L'accord du 27 janvier 2000 sur l'avenir de Mayotte ${ }^{28}$ est le premier texte à évoquer l'adoption d'une organisation juridique, économique et sociale qui se rapproche «le plus possible du droit commun », mais "adaptée à l'évolution de la société mahoraise». Son huitième point prévoit une rénovation de l'état civil et la mise en place d'un cadastre sous cinq ans, la "confortation » des droits des femmes dans la société mahoraise, et la poursuite de «la clarification $»$ du statut personnel.

Selon Sophie Blanchy et Yves Moatty, les expressions employées sont suffisamment vagues pour laisser place à des interprétations contradictoires, telles «la clarification du statut de droit local», ou «la promotion de l'identité mahoraise» qui fut comprise comme le respect de ses composantes islamiques et coutumières ${ }^{29} \ldots$ En 2010, un discours présidentiel entretiendra également ce malentendu, en évoquant une départementalisation progressive et "adaptée», sans décrire précisément ces adaptations à venir du droit coutumier. Les Mahorais qui ont voté massivement pour rester français n'ont donc que très peu pris en compte les conséquences potentielles de leur vote sur ces composantes islamiques et coutumières de leur modèle social ${ }^{30}$; et ce d'autant qu'ils savaient leur statut personnel garanti par la Constitution ${ }^{31}$.

27. Dans le titre de l'ordonnance du 8 mars 2000 (Ord. $\mathrm{n}^{\circ}$ 2000-218, 8 mars 2000, fixant les règles de détermination des nom et prénoms des personnes de statut civil de droit local applicable à Mayotte).

28. Entre le Gouvernement de la République française, le président du conseil général de Mayotte et les principaux partis de l'île (Mouvement populaire mahorais; fédération de Mayotte du Rassemblement pour la République; fédération de Mayotte du Parti socialiste).

29. S. Blanchy, Y. Moatty, «Le statut civil de droit local à Mayotte: une imposture?», Droit et société, $\mathrm{n}^{\circ} 80,2012$, p. 123.

30. E. RAlSER, «L'avenir (incertain?) du statut personnel mahorais », in F. HeRmet, Mayotte..., op.cit., p. 87.

31. Const. 1946, art. 82; Const. 1958, art. 75 (V. O. Guillaumont, «Statuts personnels et Constitution. Contribution à l'étude des articles 75 et 77 de la Constitution du 4 octobre 1958 », RRJ 2001, p. 1453). L'ordonnance du 3 juin 2010 (ratifiée par la loi du 7 décembre 2010) avait confirmé le maintien de ce statut. 
Pourtant, certains auteurs prédisent un avenir incertain ${ }^{32}$ à ce statut, ou même se demandent s'il ne constitue pas désormais une véritable «imposture $^{33}$ », et ce pour deux raisons. En premier lieu, c'est un ensemble de règles dont la place est désormais résiduelle ${ }^{34}$. Selon Élise Ralser, les «véritables sources originelles » de ce statut personnel étaient, tout autant, le droit musulman que les coutumes locales. Par référence au décret du $1^{\text {er juin } 1939}$ portant organisation de la justice indigène dans l'archipel des Comores ${ }^{35}$, elle rappelle que contrairement aux règles coutumières qui n'ont pas été codifiées, celles issues du droit musulman sont recensées dans le Minhadj at Twalibine (le guide des zélés croyants) écrit au XIII ${ }^{\mathrm{e}}$ siècle, complété par d'autres traités de rite chaféite ${ }^{36}$ : le Fath al quarib, le Ketab el tambin, et le Fath el moeni ${ }^{37}$. Cependant, les cadis peuvent aussi invoquer les coutumes locales propres à chaque île ${ }^{38}$. Comme l'explique Amir Ali, dans sa thèse, le législateur a eu «une mauvaise appréhension de la réalité des coutumes ayant cours dans l'île. Affabulé par la présence de l'Islam, il a cru à tort que le droit musulman y est fortement suivi, l'érigeant même comme source principale à travers l'institution du Minhadj comme code officiel ${ }^{39} »$. Cela rejoint l'interprétation d'une grande partie de la doctrine française qui a toujours considéré que ces règles étaient davantage de nature coutumière que confessionnelle, et qu'elles ne pourraient donc plus être modifiées par une autorité religieuse ${ }^{40}$. Le législateur a ainsi tenté de figer les règles, tout en transformant le cadre dans lequel elles pouvaient s'appliquer pour l'harmoniser avec le droit commun. Par exemple, si le statut personnel comporte des règles relatives à l'état des personnes, un véritable état civil a été institué afin de permettre le recensement des habitants mais aussi une bonne

32. E. RALSER, «L'avenir (incertain?) du statut personnel mahorais», art. cit.

33. S. Blanchy, Y. Moatty, art. cit.

34. Depuis la loi de programme pour l'outre-mer de 2003, il a été limité à l'état et à la capacité des personnes, aux régimes matrimoniaux, aux successions et aux libéralités.

35. D'après son article 6 , «les cadis jugent d'après le droit musulman et les coutumes indigènes, le Code musulman Minihadji el Talihir est le seul officiel et applicable dans l'archipel».

36. S. Blanchy, «Les textes islamiques protecteurs aux Comores: transmission et usages», in C. HAmès (dir.), Coran et talismans. Textes et pratiques magiques en milieu musulman, Paris, Karthala, 2007, p. 267.

37. Délib. n 64-12bis du 3 juin 1964 de la Chambre des députés du territoire des Comores portant réorganisation de la procédure en matière de justice musulmane.

38. Délib. n 61-16 du 17 mai 1961 de l'Assemblée territoriale des Comores relative à l'état civil à Mayotte.

39. A. Ali, L'assimilation du statut personnel mahorais, thèse dirigée par Madame Isabelle Barrière-Brousse, Faculté de droit de l'Université d'Aix-Marseille, 2016.

40. F. LuChaire, «La France d'outre-mer et la République», RFAP 2007, p. 399. 
application du droit ${ }^{41}$. Ainsi, une Commission de révision de l'état civil a établi des actes de naissance, de mariage, ou de décès pour les introduire tant dans les registres de droit commun que dans ceux de droit local. Dès lors, même les Mahorais n'ayant pas renoncé à bénéficier du statut personnel ont dû se doter d'un nom patronymique, transmissible à leurs descendants, et abandonner les règles islamiques de la nomination (un nom personnel suivi du nom personnel du père). Cette évolution a généré des incompréhensions, comme cette confusion chez certains Mahorais entre leur prénom et leur nom patronymique, en raison de l'inversion des formulaires administratifs qui placent le dernier avant le premier. Peu à peu, le statut personnel paraît vidé de sa substance.

En second lieu, il y a deux limites constitutionnelles à la garantie de ce statut. D'une part, le bénéficiaire, ou aucun de ses parents, ne doit y avoir renoncé. Certes, seule une vingtaine de Mahorais par an se soumet volontairement au statut de droit commun, mais les unions mixtes - notamment celles fréquentes entre Mahorais et Comoriens - soumettent entièrement les enfants qui en sont issus aux règles du Code civil, en réduisant d'autant le champ d'application du droit coutumier alors même que leurs deux parents partageraient une culture et des pratiques religieuses très proches de l'islam mahorais. Sans compter que les litiges entre personnes soumises au droit commun et les Mahorais bénéficiant du statut personnel ne sont pas soumis au droit local. D'autre part, le statut de droit local n'est évidemment plus garanti que dans ses dispositions qui sont conformes à l'ordre public français et aux droits et libertés fondamentales. Ainsi, le Conseil constitutionnel a admis que le législateur pouvait adopter des dispositions de nature à en faire évoluer les règles dans le but de les rendre compatibles avec les principes et droits constitutionnellement protégés ${ }^{42}$, ce qui les a modifiées substantiellement, et dans un temps très court (il aura fallu moins de dix ans ${ }^{43}$ !). Par exemple, de nombreuses modifications ont été adoptées sous le prisme du principe d'égalité. Même si l'ensemble de ces règles ne peut pas être détaillé ici, il est possible de citer l'atténuation de la différence de traitement entre les femmes et leur mari; ainsi que celle de l'autorité de leur Wali (tuteur). À partir de 2010, il a été reconnu aux femmes mahoraises le droit d'«exercer librement une profession, percevoir les gains et salaires en résultant et disposer de ceux-ci [...] administrer, obliger et aliéner» seules leurs biens

41. Par une ordonnance du 8 mai 2000.

42. Cons. const., 17 juill. 2003, $\mathrm{n}^{\circ}$ 2003-474 DC.

43. Par ex., l'ordonnance du 3 juin 2010 précise que le statut de droit local ne saurait « contrarier ou limiter les droits et libertés attachés à la qualité de citoyen français». 
personnels ${ }^{44}$. La loi du 21 juillet 2003 met fin à l'inégalité successorale entre enfants, fondée sur le sexe ou sur le caractère légitime ou naturel de la naissance. Depuis le $1^{\text {er }}$ janvier 2005, la reconnaissance de la polygamie et de la répudiation unilatérale a, elle aussi, été supprimée; mais de nombreux articles du Code civil restent inapplicables en droit local, telles les dispositions relatives au pacte civil de solidarité, ou bien le mariage pour tous $^{45}$. Si l'adoption est impossible, de même que la reconnaissance d'un enfant naturel par son père (la filiation naturelle ne crée pas de droits successoraux), une procédure en dation de nom a été instituée, aux effets très particuliers puisqu'elle vaut reconnaissance ${ }^{46}$.

Cette profonde évolution des règles du statut personnel l'a placé dans le cadre des valeurs républicaines et, plus précisément, des libertés, principes et droits constitutionnellement définis. Pourtant, dans les faits, une partie importante de la population continue à adopter des pratiques coutumières, tel le recours à un cadi pour régler un litige, alors même que la justice cadiale a été supprimée.

\subsection{LA SUPPRESSION DE LA JUSTICE CADIALE}

«L'accord sur l'avenir de Mayotte» précité stipulait expressément que le rôle des cadis serait restreint, mais il a, là encore, été mal compris par les Mahorais. Il faut dire que le recours spontané à cette institution coutumière, figure religieuse de chaque village - à la fois juge de paix, officier d'état civil et notaire - a toujours été une telle évidence pour une grande part de la population qu'il était difficile d'imaginer que les institutions républicaines pourraient s'y substituer.

Dans un premier temps, la justice cadiale a été intégrée à l'organisation judiciaire locale, mais en cessant d'être obligatoire ${ }^{47}$. Elle ne constituait plus qu'une alternative aux juridictions civiles de première instance pour les seules

44. Ord. $\mathrm{n}^{\circ}$ 2010-590, 3 juin 2010, portant dispositions relatives au statut civil de droit local applicable à Mayotte et aux juridictions compétentes pour en connaître, art. 10.

45. V. O. GoHin, « La loi et les problèmes propres à l'outre-mer», RFDA 2013, p. 979.

46. «Avec l'accord de la mère, celui qui se présente comme le père peut, par une déclaration devant l'officier de l'état civil, conférer à l'enfant, par substitution, son propre nom; cette substitution emporte reconnaissance et établissement de la filiation paternelle.

Pour l'application de l'alinéa précédent, le père et la mère doivent être des personnes de statut civil de droit local applicable à Mayotte. À défaut, la filiation ne peut être établie que dans les conditions et avec les effets prévus par le code civil» (L. n 2003-660, 22 juill. 2003 (ratif.), L. n $^{\circ}$ 2006-911, 24 juill. 2006).

47. Loi stat. de 2001, art. 61 . 
personnes relevant du statut civil de droit local, la loi du 26 mai 2004 ayant autorisé l'accès au juge de droit commun dans le cadre des conflits de toute nature entre personnes relevant du statut civil de droit local.

Dans un second temps, l'ordonnance du 3 juin 2010 a substitué de plein droit la juridiction de droit commun pour connaître des conflits entre personnes relevant du statut personnel de droit local, une décision plus conforme aux principes fondamentaux de la justice. Les cadis, désormais agents du conseil général de Mayotte, ont été véritablement dessaisis de leurs compétences juridictionnelles ${ }^{48}$ et notariales ${ }^{49}$ (comme celles de tuteurs légaux qu'ils pouvaient assumer auparavant ${ }^{50}$ ) pour ne conserver que des fonctions qu'ils exerçaient déjà en pratique: celles de médiation, de conseil et de consultation ${ }^{51}$. Cela a aussi mis fin à des techniques d'interprétation et modes de preuves traditionnels, tels ces serments décisoires devant une autorité religieuse qui étaient utilisés dans des "affaires privées», par exemple, pour écarter deux enfants naturels du partage des biens dans le cadre d'une succession ${ }^{52}$.

Or, sur ce plan encore, le modèle de l'ordre imposé pour concilier unité et diversité montre ses limites à Mayotte. Un tel modèle repose en effet sur un «droit de la subordination», qui vise à soumettre aux valeurs communes ceux qui ne les reconnaissent pas nécessairement. Encore faut-il, pour qu'elles soient véritablement appliquées, que les destinataires de ces règles juridiques se les approprient. Sinon, ils revendiqueront «leur propre conception du juste ${ }^{53}$ », et feront prévaloir un autre ordre social continuant à concurrencer le nouvel ordre juridique ${ }^{54}$. En l'absence de contestation de cet ordre social, et de recours aux autorités et juridictions incarnant l'ordre juridique, les pratiques sociales prévaudront. Par exemple, à Mayotte, l'ordonnance de 2010 précitée ne reconnaît plus que le mariage civil, y compris pour les Mahorais n'ayant pas renoncé à leur statut personnel. L'union devant le cadi est désormais un acte religieux et coutumier privé, qui ne sera pas soumis au Code civil en cas de séparation, ou de décès, pas plus qu'un cadi ne pourra légalement prononcer le divorce, enregistrer la répudiation ou superviser la succession. Conformément

48. S. Blanchy, Y. MoatTy, art. cit.

49. L. $\mathrm{n}^{\circ}$ 2010-1487, 7 déc. 2010.

50. Ibid.

51. Ord. $n^{\circ}$ 2010-590, 3 juin 2010.

52. Pour un exemple de ces affaires, antérieurement à l'adaptation des règles, V. Cass. $1^{\text {re }}$ civ., 23 mai 2006, $\mathrm{n}^{\circ}$ 05-16.809, Consorts B et X.

53. C. LE BRIS, art. cit., p. 80.

54. V. aussi C. EBERHARD, «Le droit et l'excision», in Les violences à l'encontre des femmes et le droit en France, colloque 21 et 22 nov. 2000, Univ. Paris 8. 
au droit commun, pour que des effets juridiques soient reconnus à cette union, elle devra être précédée d'un mariage civil ${ }^{55}$. Or, il est difficile de ne pas relever que les cadis conservent de fait une partie de leurs attributions en matière familiale, au risque que les juridictions civiles deviennent les chambres d'enregistrement des décisions prises en vertu du droit coutumier; du moins celles qui sont conformes au droit commun. Il est aussi très difficile de ne pas constater qu'une partie des Mahorais adopte une stratégie d'éviction, en préférant toujours officieusement s'unir de manière coutumière, y compris parfois de manière polygame ${ }^{56}$. En cas de litiges, ils recourent peu aux juridictions civiles en continuant, pour l'instant, à se tourner vers les autorités religieuses de leurs villages. Ils le font pour diverses raisons: l'habitude, la légitimité de ces institutions et la confiance qu'ils placent en elles ${ }^{57}$, voire même des considérations financières. Certes, solliciter les cadis peut s'inscrire dans le cadre de leurs nouvelles fonctions de conciliation et de médiation, mais un problème peut se poser lorsqu'une véritable portée normative est donnée aux conseils reçus par leurs destinataires. Cette stratégie d'éviction, parce qu'elle entraîne un recours aux autorités coutumières risque de faire subsister de facto des situations, certes légitimées par l'ordre social, mais illégales; sans que les droits subjectifs des personnes impliquées dans ces situations ne puissent toujours être protégés, notamment lorsqu'elles sont vulnérables. Et puis, si les cadis et les autres autorités religieuses de l'île ne doivent se prononcer qu'en matière civile, des «sanctions » socialement acceptées peuvent encore être appliquées à des actes ayant violé le droit pénal coutumier, sans qu'une autorité publique en ait toujours connaissance.

Ces limites à l'application du droit commun peuvent être considérées comme l'expression de la nécessaire période d'adaptation de Mayotte au cadre constitutionnel qui a été posé. Reste donc toujours la question de l'incorporation des valeurs républicaines à l'ordre social. Pour ce faire, depuis la départementalisation, la voie empruntée n'est plus celle de l'ordre imposé, mais celle de l'ordre négocié.

55. En vertu de l'article 433-21 du Code pénal, toute autorité religieuse, qui procéderait, de manière habituelle, aux cérémonies religieuses de mariage, sans s'assurer qu'un mariage civil a bien été célébré, risquerait une peine de six mois d'emprisonnement et de 7500 euros d'amende.

56. D'après l'enquête de l'INSEE, Migrations, natalité et solidarités familiales, en 2017, un homme sur dix était toujours polygame. Ils étaient $13 \%$ en 1991, ce qui montre une baisse peu marquée des pratiques (V. «La polygamie à Mayotte: une pratique rejetée par une majorité de femmes... un peu moins par les hommes», Le journal de Mayotte, 13 mars 2017).

57. V. infra. 


\section{L'ORDRE NÉGOCIÉ DÉSORMAIS PRIVILÉGIÉ}

L'ordre négocié se traduit essentiellement par la formation aux valeurs de la République (2.1). Néanmoins, selon Catherine Le Bris, le risque de cet ordre négocié est la déliquescence des valeurs partagées «dans un univers des différences ${ }^{58} »$; ce risque ne pouvant être évité que par une réelle application des principes républicains (2.2).

\subsection{L'INTÉGRATION DE MAYOTTE DANS LA FORMATION AUX VALEURS DE LA RÉPUBLIQUE}

D'un point de vue juridique, et non plus seulement d'un point de vue politique, la conciliation entre des valeurs mahoraises marquées par la singularité et les valeurs républicaines à vocation universelle a été un sujet très peu abordé par le législateur; les débats doctrinaux et parlementaires se concentrant sur les enjeux institutionnels. Cette question était d'autant moins discutée que, s'il existe bien quelques mouvements indépendantistes organisés à Mayotte, aucun n'a été jusqu'à présent fondé sur des considérations religieuses ou même sur une contestation des valeurs de la République ${ }^{59}$.

Il faut préciser qu'il existait un postulat selon lequel les électeurs mahorais ayant exprimé leur volonté de rester dans la République entendaient aussi en adopter les valeurs. Ainsi, Olivier Gohin écrivait-il en 2009 que «les Mahorais ou leurs représentants nationaux et locaux ont fait preuve, par le passé, de suffisamment de constance et de maturité pour envisager sereinement l'avenir, sachant qu'au bout du compte, pour avoir été vigilants, ils resteront libres, dans cette égalité qu'ils ont voulue et dans cette fraternité qu'ils ont exprimée, par le biais du suffrage universel ${ }^{60} »$. En 2014, c'était un rapport devant le Conseil supérieur de la fonction publique qui affirmait que «l'attachement de Mayotte à la France et aux valeurs de la République n'est plus à démontrer. Mayotte envers et contre tout et tous a voulu rester française ${ }^{61}$ ». Il est vrai que des responsables politiques locaux,

58. V. aussi E. LE Roy, «L'ordre négocié. À propos d'un concept en émergence », in P. GÉRARD, F. Ost, M. Van de Kerchove (dir.), Droit négocié, droit imposé?, Bruxelles, Facultés universitaires Saint-Louis, 1996, p. 341.

59. V. I. Mamaye, «Échec des mouvements unionistes et indépendantistes à Mayotte: de leur formation à leur négation (1958-2011)», Mouvements, n 91, 2017, p. 160.

60. O. Gohin, «Mayotte: la longue marche...», art. cit.

61. B. Collignon, Mayotte, «les oubliés de la République», Rapport au CSFP, Ministère de l'Intérieur, 2014, p. 2. 
comme le député Mansour Kamardine, ont placé ces différentes étapes sous les auspices républicains ${ }^{62}$. Toujours dans cette même conception, un autre rapport de 2012 - sénatorial cette fois-ci - regrettait un attachement déçu, car selon lui, malgré une «volonté maintes fois réaffirmée, force est de constater que l'attachement des Mahorais aux valeurs de notre pays a tardé à recevoir la réponse qu'il pouvait légitimement attendre ${ }^{63} »$. Sur l'île, ce postulat d'une adhésion de facto aux valeurs républicaines se traduit dans les discours des autorités publiques par la proclamation régulière de points communs entre la culture mahoraise et la liberté, l'égalité, la fraternité ou la laïcité.

Or il est possible d'avoir deux conceptions des valeurs républicaines: l'une défensive, l'autre plus pédagogique. Dans la première acception, les valeurs républicaines seront perçues comme un idéal d'universalité mises «à l'épreuve par des revendications particulières ${ }^{64} »$. Dans la seconde conception, les valeurs républicaines se diffuseront par l'explication et l'enseignement ${ }^{65}$. C'est cette seconde interprétation qui est privilégiée à Mayotte. À partir de 2015, comme en métropole, les religieux, et les "professionnels en contact direct avec les publics ${ }^{66} »$ ont été les destinataires de la mise en place d'actions nationales de formations aux valeurs de la République, à commencer par les agents publics, et salariés des associations de la ville, de la jeunesse et des sports. C'est notamment le cas du plan de formation conçu sous l'égide du Commissariat général à l'égalité des territoires (CGET), en collaboration avec le Centre national de la fonction publique territoriale (CNFPT). En décembre 2016, la direction de la Jeunesse, des Sports et de la Cohésion Sociale (DJSCS) de Mayotte a ainsi constitué une commission départementale pour établir un «schéma de développement des valeurs de la République». La même année, toujours sur un modèle similaire à celui adopté en métropole, le Centre universitaire de Mayotte a mis en place le diplôme universitaire «Islam et valeurs de la République» (qui, depuis, s'intitule «Religions et valeurs de la République»), présenté par le préfet, d'alors, comme devant permettre de s'«interroger sur les valeurs de la France, de la République et de l'islam [...] pour que Mayotte, dans son appropriation de la République, se

62. M. Kamardine, Discours de la République pour Mayotte, Saint-Denis, Orphie, 2007.

63. J.-P. Sueur, Ch. Cointat, F. Desplan, Mayotte: un nouveau département confronté à de lourds défis, Rapp. Sénat, nº 675, 2012.

64. France. Haut Conseil à l’intégration, Études et intégration: faire connaître les valeurs de la République, Paris, La Documentation française, 2009, p. 30.

65. Le même rapport explique plus loin qu'il convient d'expliquer ces principes républicains afin «de faire comprendre leur sens et de les faire partager» (ibid.).

66. Selon les termes employés lors du Comité interministériel à l'égalité et à la citoyenneté du 6 mars 2015. 
dise: j'ai un miroir... je m'y reconnais d'une manière ou autre ${ }^{67} »$. Le public visé était initialement les cadis et les aumôniers, même si d'autres professionnels les ont rejoints pour être formés dans des matières telles que: la place de Mayotte dans la République, la liberté, l'égalité, la fraternité, le service public, la langue française, «la vie en commun, les valeurs de l'enseignement et de la santé, le travail, l'enfance, la jeunesse, le droit des femmes ${ }^{68}{ }$.

Cette conception de la diffusion des valeurs républicaines, centrée sur l'éducation, correspond davantage à la définition théorique d'une valeur comme la qualité «d'une chose ou d'une action, en vertu de laquelle elles méritent de l'estime ou font l'objet d'un désir ${ }^{69}{ }$. Elle permet alors de laisser deux options au destinataire de cet enseignement: soit la pleine adhésion, c'est-à-dire le partage des valeurs républicaines par l'abandon des siennes, un processus qui peut être d'autant plus long qu'il résulte de l'imprégnation par de nouveaux codes sociaux ${ }^{70}$; soit le respect, en trouvant une forme de conciliation entre les valeurs républicaines et ses propres comportements quotidiens ou pratiques sociales. Cependant, le risque de traiter tous les territoires de manière identique est de ne pas prendre en compte leurs singularités, alors même que le statut constitutionnel de Mayotte l'impose, surtout depuis l'article 72-3 qui dispose que «La République reconnaît, au sein du peuple français, les populations d'outre-mer, dans un idéal commun de liberté, d'égalité et de fraternité».

\subsection{L'APPLICATION DU PRINCIPE DE LAÏCITÉ}

Les principes constitutionnels sont eux-mêmes porteurs de valeurs ${ }^{71}$. De leur application dépend donc aussi l'adhésion à ces mêmes valeurs. Toutefois, Sophie Blanchy et Yves Moatty constataient dès 2012 qu'avec l'intégration institutionnelle plus poussée de Mayotte, "on s'était attaqué à un système [axiologique] bien incorporé» et qu'il aurait donc fallu s'assurer préalablement de la transition vers les nouvelles valeurs ${ }^{72}$ avant de modifier le droit commun. Effectivement, la départementalisation était avant tout présentée comme un avantage d'un point de vue économique et social, sans que n'ait

67. Propos recueillis par E. TusEvo, pour le site La $1^{\text {re }}$ France TV info, 8 sept. 2015.

68. Ibid.

69. J.-M. Delgado Ocando et A.-J. Arnaud, «Valeur», in A.-J. Arnaud (dir.), Dictionnaire encyclopédique de théorie et de sociologie du droit, Paris, LGDJ, 1993, p. 633.

70. Haut Conseil a l'intégration, op. cit.

71. H. Fulchiron, M. Philip-Gay, «La laïcité: un principe, des valeurs», D. 2015, p. 274.

72. S. Blanchy, Y. Moatty, art. cit. 
été véritablement pensé ce moment où les valeurs républicaines, venues de si loin géographiquement et culturellement, viendraient réinterroger les valeurs locales, dont l'île s'est toujours employée à conserver les particularités. Or Aimé Césaire décrivait «deux manières de se perdre: par ségrégation murée dans le particulier ou par dilution dans l'universel». Tout l'enjeu pour Mayotte ${ }^{73}$ est précisément de ne pas s'enterrer dans ce "particularisme étroit », ni de se perdre dans l'« universalisme décharné » que décrivait Césaire $^{74}$. L'application des principes fondamentaux de la République vise à éviter ces deux écueils.

Premièrement, il s'agit d'éviter le «particularisme étroit» craint par Césaire. Par exemple, la laïcité, cette «forme française de la liberté religieuse ${ }^{75}$ » peut permettre de légitimer la nécessaire séparation de fait entre les normes juridiques et les normes sociales (dont les normes religieuses), qui, même si elles sont en rapport les unes avec les autres, devront nécessairement avoir un mode de validité strictement différent ${ }^{76}$. Elle le peut d'autant plus que Mayotte fait partie des collectivités soumises au régime juridique défini par le décret Mandel du 16 janvier 1939, et pour lesquelles la loi de 1905 séparant les Églises et l'État ne s'applique pas dans son intégralitée ${ }^{77}$. Ce décret ne s'oppose donc pas à ce que le département finance des dépenses relatives aux cultes, à condition de respecter l'égalité entre les différentes convictions, la neutralité des collectivités territoriales; et sous réserve qu'il existe un motif d'intérêt général pour le territoire concerné ${ }^{78}$. D'autres modèles, par exemple celui de La Réunion (où pourtant la loi de 1905 s'applique), démontrent qu'il est possible de concilier la neutralité des personnes publiques avec une importante visibilité religieuse dans la sphère sociale. La laïcité n'a pas pour objet de «neutraliser» l'espace public, ni les personnes privées, sauf exceptions définies par la loi. Être précis sur la distinction des espaces et des personnes soumises à des obligations en vertu du principe de laïcité est certainement l'une des conditions pour éviter le risque de déliquescence des valeurs déjà évoqué. Cela est d'autant plus important, que - dans les faits les difficultés de mise en ouvre du principe de laïcité à Mayotte concernent

73. V. F. Hermet, Mayotte..., op. cit.

74. Lettre de démission d'Aimé Césaire adressée à Maurice Thorez, 24 oct. 1956.

75. Y. Gaudemet, «La laïcité, forme française de la liberté religieuse», Administration $\mathcal{E}$ Éducation, vol. 148, 2015, p. 111.

76. J.-Ch. Le Coustumer., «La norme et l'exception. Réflexion sur les rapports du droit avec la réalité», CRDF, n 6, 2007, p. 19.

77. V. CE, avis, 20 mai 2010, $\mathrm{n}^{\circ} 383887$.

78. CE, 16 mars 2005, n² 265560, Min. Outre-mer c. Polynésie française. 
précisément la neutralité des agents de la fonction publique, et dans une moindre mesure, l'application de la loi du 15 mars 2004 encadrant le port de signes ou de tenues manifestant une appartenance religieuse dans les écoles, collèges et lycées publics. Il faut préciser qu'initialement, le premier projet de circulaire d'application de cette dernière prévoyait que cette loi de 2004 n'avait pas «pour objet d'interdire les tenues traditionnelles là où elles [avaient] été jugées jusqu'ici compatibles avec le principe de laïcité et le bon fonctionnement du service public de l'enseignement ${ }^{79}$ ", ce qui aurait permis de reconnaître la singularité des tenues mahoraises, notamment féminines. Bien que cette précision ait été retirée de la circulaire finalement diffusée, et parce que ces tenues ne sont généralement pas perçues comme religieuses, la confusion subsiste dans certains établissements scolaires publics.

Toutefois, les dirigeants politiques locaux présentent le principe de laïcité comme un moyen de protéger l'islam mahorais contre le fondamentalisme et les «interprétations de l'extérieur», tout en permettant le pluralisme convictionnel. Il y a déjà une prise en compte par le droit local des autres confessions, comme le culte catholique, pratiqué essentiellement par les métropolitains. Depuis 1940, il s'est organisé en mission et dépend du diocèse des Comores, et non pas de celui de la Réunion (le Vatican s'alignant ainsi sur les résolutions de l'assemblée générale de l'ONU) ${ }^{80}$. En outre, la liberté religieuse, présentée par le Conseil d'État comme une déclinaison du principe de laïcité ${ }^{81}$ peut juridiquement être conçue comme figurant "parmi les éléments les plus essentiels de l'identité des croyants et de leur conception de la vie, mais elle est aussi un bien précieux pour les athées, les agnostiques, les sceptiques ou les indifférents ${ }^{82} »$. Elle permet donc également de protéger par le droit les pensées minoritaires existant à Mayotte, ce qui répond aux aspirations d'une partie de la jeunesse. Comme l'exprime Éléonore Cadou,

«Nombreux sont les adolescents déchirés entre culture traditionnelle et culture occidentale, en situation de conflit majeur avec leurs parents, et l'accélération des mutations de la société mahoraise ne rend pas optimiste quant au développement d'actes de délinquance. Pour autant, le processus de mise à niveau des références juridiques de l'archipel porte déjà ses premiers fruits, et on ne peut que se réjouir que les termes

79. JO, 3 mai 2004, p. 902.

80. France. Conseil d’État, Un siècle de laïcité, Paris, La Documentation française, 2004, p. 271.

81. «La laïcité française doit, à tout le moins, se décliner en trois principes: ceux de neutralité de l'État, de liberté religieuse et de respect du pluralisme» (Ibid., p. 272).

82. CEDH, 25 mai 1993, n 14307/88, Kokkinakis c. Grèce. 
d'égalité et de liberté prennent une signification plus concrète au sein des familles mahoraises soumises au droit local ${ }^{83} »$.

Deuxièmement, pour éviter l'« universalisme décharné» que décrivait Césaire $^{84}$, il est possible d'identifier deux «modes de repères dans cette société en pleine mutation ${ }^{85}$ », sur lesquels l'application des principes fondamentaux pourrait s'appuyer. Le premier de ces repères est le cadi. Une enquête publiée en mars 2017 faisait apparaître combien cette institution restait importante pour une très grande majorité des sondés; dont $44 \%$ reconnaissaient toujours y faire appel, selon les cas, pour «des conseils», ou «comme référent important dans [leur] vie ${ }^{86} »$. Ces résultats laissaient apparaître que la perte de son rôle juridique est très majoritairement acceptée, mais qu'il semble toujours y avoir un attachement moral à sa fonction sociale fondée sur «sa sagesse». Depuis mai 2016, les vingt et un cadis ont été intégrés à la Direction départementale de la médiation, de la conciliation, et du règlement des conflits, afin de leur permettre de devenir de véritables médiateurs sociaux, ce qui devrait les réinstitutionnaliser, dans des fonctions plus coutumières que religieuses, et donc permettre qu'ils soient l'un des supports de l'appropriation des valeurs, à condition de renforcer encore leur formation.

Le second repère est certainement la place des femmes dans la société mahoraise. Dans son discours de 2001, le secrétaire d'État à l'Outre-mer Christian Paul les décrivait déjà comme «l'élément stabilisateur de la société mahoraise. Elles sont propriétaires de leur maison, leurs maris ne le sont pas. Les coutumes africaines tendent ainsi à neutraliser certains effets de la loi islamique ${ }^{87} »$. Cependant, le principe matrilinéaire et celui de la matrilocalité n'empêchaient pas une discrimination coutumière, notamment dans le cadre du mariage et des successions. Les modifications du statut civil de droit local leur ont permis d'être plus autonomes financièrement. En outre, depuis 2000, elles sont entrées massivement dans les dix-sept conseils municipaux de Mayotte, étant plus de deux cent cinquante à être désormais élues,

83. E. CADOU, «Le statut de l'enfant dans l'Océan indien: l'enfant mahorais », RID comp. 2005, p. 342.

84. Lettre de démission d'Aimé Césaire adressée à Maurice Thorez, 24 oct. 1956.

85. V. G. VAuthiER, «Enquête: Mayotte roule-t-elle toujours pour les cadis? », Mayotte Hebdo, 9 mars 2017.

86. Ibid.

87. Discours de M. Christian Paul, secrétaire d'État à l'Outre-mer, sur la situation juridique, économique et sociale de Mayotte, le projet de loi visant à fixer le statut de l'île et les efforts déployés par l'État en vue de son développement économique et social, Paris, 4 avril 2001. 
ce qui constitue «une révolution tranquille ${ }^{88} »$. Leurs tenues traditionnelles (salouva), très colorées, et leur châle (kishali) porté sur les épaules ou sur les cheveux pourraient même interroger à leur tour le droit français de la laícité: en juin 2017, la députée de Mayotte nouvellement élue apparaissait sur sa photo officielle avec un kishali posé sur sa tête. Alors qu'elle prenait soin de le distinguer d'une tenue religieuse, et de ne le porter que sur les épaules lorsqu'elle siégeait en commission ou dans l'hémicycle, sa présence à l'Assemblée contribue certainement à expliquer la modification de l'instruction générale du Bureau relative à la tenue des députés en séance, interdisant désormais dans l'hémicycle, notamment «le port de tout signe religieux ostensible ${ }^{89} »$. Reste toutefois à parier que cette disposition, juridiquement contestable, ne lui serait pas applicable au regard des précautions prises par l'élue.

En conclusion, «la nationalité n'est pas une garantie de valeurs "communes", l'étrange pouvant être le fait du national. Bien souvent, c'est lorsque ces valeurs sont contestées, confrontées à l'altérité, c'est-à-dire en cas de différend, qu'elles se révèlent ${ }^{90} »$. Or, ceux qui pensaient précisément voir en Mayotte un lieu de confrontation entre ces valeurs communes et l'islam se sont lourdement trompés, de même que l'appropriation semble en marche à condition d'étendre la formation sur les valeurs de la République. Cette constatation n'empêche tout de même pas de regretter que l'État ne fasse pas davantage vivre ces valeurs à Mayotte, notamment celle de la dignité humaine, en accélérant encore le développement de cette île au très fort potentiel économique et le co-développement en faveur des Comores.

88. Ibid.

89. Instruction générale du bureau de l'Assemblée nationale, art. 9 «Tenue en séance».

90. C. LE BRIS, art. cit., p. 79. 